\title{
Controlling the Second Harmonic in a Phase-Matched Negative-Index Metamaterial
}

\author{
Alec Rose, Da Huang, and David R. Smith \\ Center for Metamaterials and Integrated Plasmonics, Duke University, Durham, North Carolina 27708, USA
}

(Received 31 May 2011; revised manuscript received 17 June 2011; published 1 August 2011)

\begin{abstract}
Nonlinear metamaterials have been predicted to support new and exciting domains in the manipulation of light, including novel phase-matching schemes for wave mixing. Most notable is the so-called nonlinear-optical mirror, in which a nonlinear negative-index medium emits the generated frequency towards the source of the pump. In this Letter, we experimentally demonstrate the nonlinear-optical mirror effect in a bulk negative-index nonlinear metamaterial, along with two other novel phase-matching configurations, utilizing periodic poling to switch between the three phase-matching domains.
\end{abstract}

DOI: 10.1103/PhysRevLett.107.063902

PACS numbers: 42.65.Ky, 78.67.Pt

Nonlinear metamaterials (NLMMs) represent the pinnacle of designer electromagnetic materials, allowing one to manipulate light based on direction, polarization, frequency, and intensity. In particular, these artificial mediums have the potential to make nonlinear-optical phenomena accessible at low powers and in novel and exciting configurations $[1,2]$. While a wide range of nonlinear processes have already been demonstrated in NLMMs, including frequency generation [3-5], parametric amplification [6,7], and bistability [8,9], the setups have been constrained to subwavelength interaction lengths for fear of the destructive effects of phase mismatch. As such, the conversion efficiencies achieved are only a fraction of their potential. However, phase matching (PM) is an exciting subject that involves the entire set of linear and nonlinear properties, bringing to bear the full capabilities of NLMMs to manipulate light at will. This claim has been supported by several theoretical studies on NLMMs, most notably the prediction of a nonlinear-optical mirror that generates the second harmonic (SH) back towards the fundamental frequency (FF) source $[6,10,11]$, but experimental verification of the remarkable PM effects achievable in bulk NLMMs has been lacking.

In this Letter, we experimentally demonstrate three unique PM configurations for second-harmonic generation (SHG) in a metallic waveguide loaded with a magnetic NLMM at microwave frequencies. The three configurations are reflected SH PM in a negative-index band, transmitted SH quasiphase matching (QPM), and simultaneous QPM of both the reflected and transmitted SHs near a zeroindex band. The resulting SH spectrums, showing strong PM-induced enhancements, are supported qualitatively by nonlinear transfer matrix method (TMM) calculations on a homogeneous slab with equivalent effective properties.

The NLMM under investigation in this Letter is the varactor-loaded split-ring resonator (VLSRR), the canonical subject of numerous recent studies. The constituent unit cell of this NLMM is composed of a $17 \mu \mathrm{m}$ thick, $0.75 \mathrm{~mm}$ wide copper ring printed on a $0.2 \mathrm{~mm}$ FR4 printed circuit board substrate. The $1 \mathrm{~mm}$ gap is loaded with a Skyworks
SMV1231 varactor diode, whose capacitance varies with applied voltage according to $C\left(V_{D}\right)=C_{0}\left(1-V_{D} / V_{p}\right)^{M}$, where $V_{D}$ is the voltage across the diode, $C_{0}=2.35 \mathrm{pF}$ is the zero-voltage capacitance, $V_{p}=1.5 \mathrm{~V}$ is the intrinsic potential, and $M=0.8$ is the gradient coefficient [12]. Our bulk material, displayed in Fig. 1, consists of 16 layers (24 $\mathrm{cm}$ in length) of VLSRRs placed in an aluminum waveguide with cross section $15 \mathrm{~cm} \times 1.5 \mathrm{~cm}$, completely filling the waveguide in the transverse direction, and arranged in a square lattice with $1.5 \mathrm{~cm}$ spacings. For the purpose of periodic poling, the VLSRR sample is divided into four identical sections that can be reoriented independently. These dimensions ensure that the resonance frequency of the VLSRR is below the cutoff frequency of the waveguide's lowest-order mode. As such, the NLMM loaded waveguide is expected to support a backward mode at frequencies where the VLSRR's permeability is negative, that is, a mode whose phase velocity is opposite to the direction of power flow [13-15]. In addition, near the cutoff frequency, the wave vector of this mode vanishes, yielding a second region of interest for PM.

The linear properties of the VLSRRs were characterized in a transmission line setup by measuring the transmittance $\left(\left|S_{21}\right|\right)$ of a single layer and fitting to known property models [16], shown in Fig. 2(a). We find our VLSRR medium to be well-described by a constant permittivity

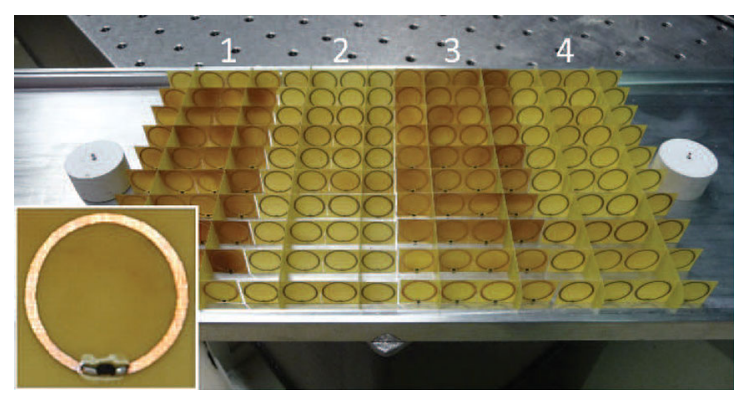

FIG. 1 (color online). Photograph of the waveguide with the top removed, loaded with four identical sections of VLSRRs. The inset shows an enlarged view of the NLMM unit cell. 
$\epsilon_{y}(\omega)=2.2 \epsilon_{0}$ and a permeability given by the Lorentz oscillatorlike formula

$$
\mu_{x}(\omega)=\mu_{0}\left(1+\frac{F \omega^{2}}{\omega_{0}^{2}-i \gamma \omega-\omega^{2}}\right)
$$

where $F=0.22$ is the oscillator strength, $\omega_{0}=$ $2 \pi \times 608 \mathrm{MHz}$ is the resonance frequency, and $\gamma=2 \pi \times$ $14 \mathrm{MHz}$ is the damping coefficient. The permeability is assumed to be that of free space along the other axes.

Subsequently, we measured the SHG output from this setup. Using the nonlinear TMM retrieval method [16-18], we extracted the second-order magnetic nonlinear susceptibility from the SH spectrum [shown in Fig. 2(b)]. The result was then fitted to an effective medium model obtained via a perturbative circuit analysis [19]:

$$
\chi_{m}^{(2)}(2 \omega ; \omega)=\frac{-(2 i) a F \omega_{0}^{4} A \mu_{0} \omega^{3}}{\left(\omega_{0}^{2}-i \gamma \omega-\omega^{2}\right)^{2}\left(\omega_{0}^{2}-2 i \gamma \omega-4 \omega^{2}\right)},
$$

where $a= \pm 0.198 \mathrm{~V}^{-1}$ is the fitted strength of the nonlinearity and $A=113 \mathrm{~mm}^{2}$ is the ring's area. The sign of $a$ is determined by the orientation of the varactor diode in the VLSRR, reflecting the fact that reversing the diode direction is analogous to reversing the orientation of the nonlinear metacrystal, and thus is expected, by symmetry arguments, to induce a $\pi$ phase shift in the even-order nonlinearities. Note that the magnitude of $a$ is close to the predicted value of $\frac{M}{2 V_{p}}=0.267 \mathrm{~V}^{-1}$ [19].

The longitudinal wave vector of the $\mathrm{TE}_{10}$ mode of the metallic waveguide is modeled by

$$
k_{z}(\omega)= \pm \omega \sqrt{\mu_{x}\left[\epsilon_{y}\left(1-\frac{\omega_{c}^{2}}{\omega^{2}}\right)\right]},
$$

where $\omega_{c}=\pi /\left(b \sqrt{\epsilon_{y} \mu_{0}}\right)=2 \pi \times 674 \mathrm{MHz}$ is the cutoff frequency and $b=15 \mathrm{~cm}$ is the waveguide width. As in typical negative-index media, the negative sign is chosen when both terms in the square root are negative, i.e., when

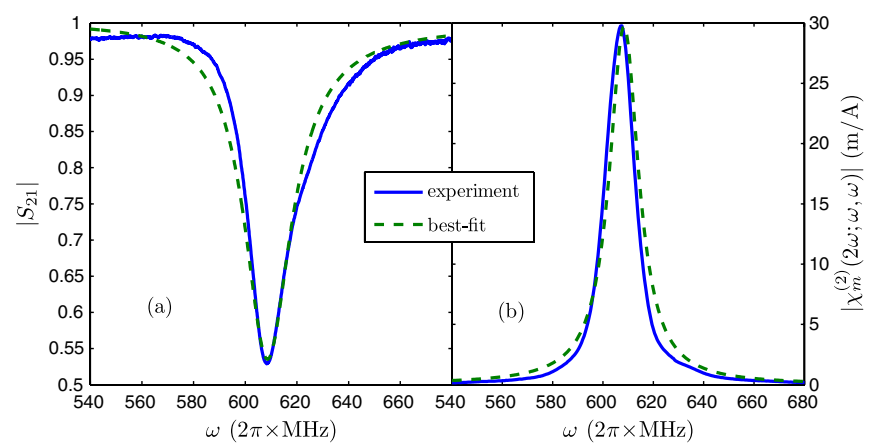

FIG. 2 (color online). Plot of the magnitude of the retrieved (solid blue curve) and fitted (dashed green curve) transmittance (a) and second-order susceptibility (b) for a single-layer VLSRR slab. $\mu_{x}<0$ and $\epsilon_{y}\left(1-\frac{\omega_{c}^{2}}{\omega^{2}}\right)<0$. The former occurs just above the resonance frequency of the VLSRR, while the latter requires operation of the waveguide below the cutoff, so that we can expect a backward wave to exist for frequencies of roughly $615 \mathrm{MHz}<\omega / 2 \pi<674 \mathrm{MHz}$. Furthermore, for a FF below cutoff, it can be shown that only the $\mathrm{TE}_{10}$ mode will propagate at the $\mathrm{SH}$, simplifying our analysis. For coupling between the $\mathrm{TE}_{10}$ modes at the $\mathrm{FF}$ and $\mathrm{SH}$, we obtain an overlap integral of $\Gamma \approx 1.2$.

The PM effects in our VLSRR loaded waveguide can be examined by employing the above experimentally fitted models to calculate the SHG phase mismatch and coherence lengths. For example, the phase mismatch for SHG in a homogeneous medium is given by

$$
\Delta k= \pm k_{z}(2 \omega)-2 k_{z}(\omega) \text {, }
$$

where it is assumed that the energy of the FF wave propagates in the positive $z$ direction and the \pm refers to a positively (transmitted) or negatively (reflected) propagating $\mathrm{SH}$ wave. Thus, assuming the $\mathrm{SH}$ is far from resonance, it is clear that a forward wave $\left[k_{z}(\omega)>0\right]$ at the FF will tend to phase match with a transmitted $\mathrm{SH}$ wave, as is seen in conventional nonlinear optics. A backward wave $\left[k_{z}(\omega)<0\right]$ at the FF, on the other hand, will tend to phase match with a reflected $\mathrm{SH}$ wave, as predicted earlier $[6,10,11]$. This odd behavior can be seen more clearly by computing the coherence lengths $L_{\mathrm{coh}}=\frac{2 \pi}{\Delta k}$, shown in Fig. 3 using Eqs. (3) and (4). As expected, the coherence length for the transmitted SH is small (less than the interaction length), while the coherence length for the reflected $\mathrm{SH}$ is much larger throughout the negative-index band. The reflected PM region, shown in gray in Fig. 3, represents the frequency range in which our NLMM acts as the nonlinearoptical mirror of Refs. [6,11], with the vast majority of the $\mathrm{SH}$ power traveling opposite to the FF.

In an inhomogeneous medium with some spatial periodicity, however, the PM condition is rewritten to include

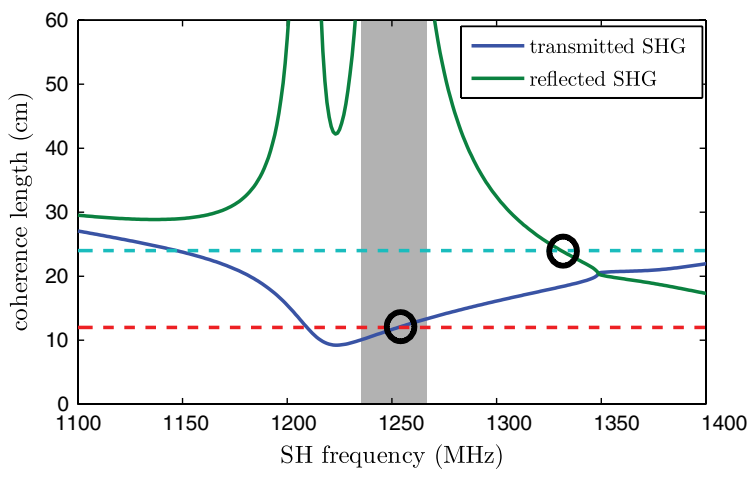

FIG. 3 (color online). Plot of the calculated coherence lengths for transmitted (blue curve) and reflected (green curve) SHG in the VLSRR loaded waveguide. The dashed lines at 12 and $24 \mathrm{~cm}$ correspond to the two poling periods used in this Letter, while the gray band indicates nonlinear-optical mirror behavior. 
the corresponding reciprocal lattice vector. In periodically poled QPM, for example, the sign of the nonlinear susceptibility is reversed in neighboring layers with a period of $\Lambda$. QPM then requires

$$
\pm k_{z}(2 \omega)=2 k_{z}(\omega)+2 m \pi / \Lambda
$$

or, equivalently, $\Lambda=m L_{\text {coh }}$, where $m$ is any integer. As mentioned earlier, poling in our system can be achieved by simple reorientation of appropriate sections of VLSRRs, analogous to domain engineering. Thus, by taking $m=1$ in Fig. 3, the intersection of the dashed lines (representing poling periods of 12 and $24 \mathrm{~cm}$ ) with the coherence length curves indicates the frequencies at which Eq. (5) is expected to be satisfied, resulting in enhanced SHG output for the respective configuration.

Furthermore, we see that something interesting happens as the FF approaches the waveguide cutoff. Near this frequency, $k_{z}(\omega)$ vanishes. Thus, Eq. (5) can be satisfied for both the reflected and transmitted modes by the same poling period. This is indicated in Fig. 3 as the point where the reflected and transmitted modes cross, corresponding to where the FF passes through the cutoff frequency. Thus, for poling periods in the proximity of this coherence length, we expect to see QPM enhancement of both the reflected and transmitted $\mathrm{SH}$ waves.

In order to inject and measure the FF and $\mathrm{SH}$ waves, respectively, the inner conductors (probes) of two coaxial cables are inserted into the waveguide on either side of the VLSRR sample. The probes are positioned $1.5 \mathrm{~cm}$ from the sample and covered with an alumina shell to reduce the impedance mismatch between the coaxial cable and the waveguide. Rectangular aluminum slabs with a cross section exactly equal to the waveguide are placed at a variable distance from the probes. By manually optimizing the distance between these back walls and the probes, we are able to reduce reflections at the coaxial-waveguide interface to less than $0.75 \mathrm{~dB}$ at the $\mathrm{SH}$ frequencies of interest, preserving the directionality of the generated $\mathrm{SH}$ wave. The FF, however, does not need to be matched because the much larger material losses in the FF range prevent significant back-reflection inside the sample. In other words, the vast majority of the incident FF power that is able to enter the sample travels in the forward direction and is absorbed by the NLMM before reaching the second interface, preserving the directionality of the FF wave. Thus, by pumping through one probe at the FF and measuring the SH power at both probes, we are able to simultaneously investigate the reflected and transmitted SHG in the VLSRR loaded waveguide. For clarity, a diagram of the experimental apparatus is shown in Fig. 4. An Agilent PNA-X N5245A network analyzer is used as both the source and receiver.

In order to validate our experimental results and interpretations, we employ nonlinear TMM calculations on a homogeneous slab with equivalent effective properties to

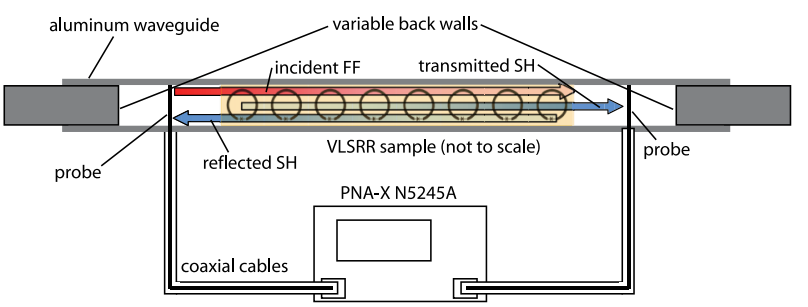

FIG. 4 (color online). Diagram of the experimental setup employed to measure the SH spectrums, depicting a cross section of the VLSRR loaded aluminum waveguide.

the VLSRR loaded waveguide, sandwiched between semiinfinite slabs of vacuum, assuming the nondepleted pump approximation $[17,18]$. The power incident on the sample at the FF is approximated by $P_{i}=P_{0}-P_{r}$, where $P_{r}$ is the reflected power measured in the experiment and $P_{0}$ is the source's output power. The losses before and after the sample are assumed to be $0.75 \mathrm{~dB}$ at all frequencies. Although this system is clearly a rough approximation of the actual experimental setup, qualitative agreement is still expected, especially regarding the location and shape of the PM enhancements.

First, we investigate PM of the reflected SHG in a negative-index band. All four VLSRR sections are aligned to simulate a homogeneous nonlinear medium. The source is swept from 600 to $650 \mathrm{MHz}$ with an output power of $-5 \mathrm{dBm}$. The resulting transmitted and reflected SH spectrums are plotted in Fig. 5. The plot shows significant enhancement in the SHG of the reflected wave over the transmitted and is supported qualitatively by the inset showing the TMM result. Furthermore, the peak frequency of $1255 \mathrm{MHz}$ lies well within the range predicted by the coherence length calculation of Fig. 3. These results confirm the referenced theoretical studies, and in this configuration our NLMM can be considered a nonlinear-optical

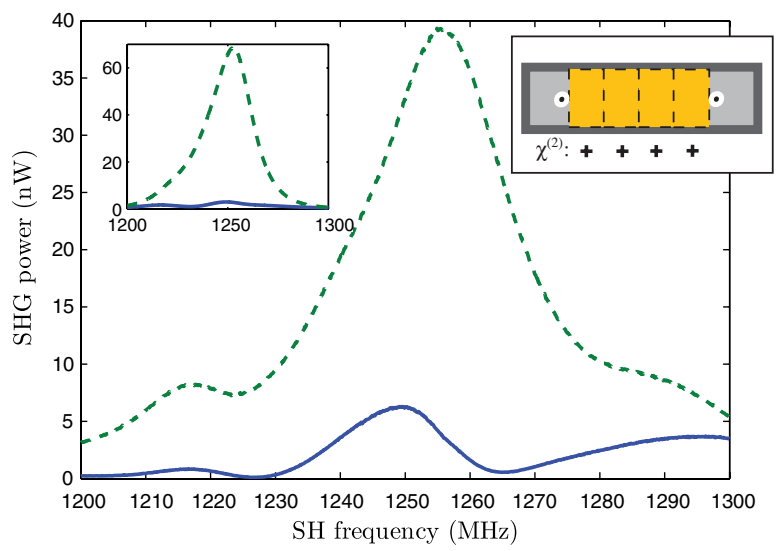

FIG. 5 (color online). Comparison of the transmitted (solid blue curve) and reflected (dashed green curve) SHG powers when all sections are aligned. The right inset depicts the experimental configuration, and the left inset shows the TMM calculation. 


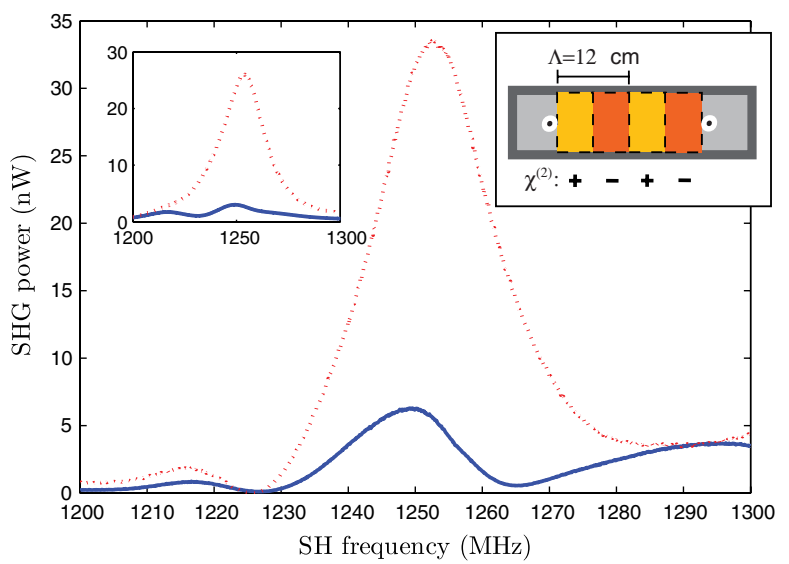

FIG. 6 (color online). Comparison of the transmitted SHG power when the varactors are aligned (solid blue curve) and when they are periodically poled with $\Lambda=12 \mathrm{~cm}$ (dotted red curve).

mirror. For the network analyzer's maximum output, we were able to display a conversion efficiency as high as $1.5 \%$.

In the next experiment, the previous configuration is retained in all ways except that sections 2 and 4 are physically rotated by $180^{\circ}$, effectively producing a periodically poled nonlinear crystal with $\Lambda=12 \mathrm{~cm}$. The transmitted SH spectrum of this QPM setup is compared to that of the original in Fig. 6. The transmitted SH power is greatly increased due to QPM, with the peak frequency of $1253 \mathrm{MHz}$ in excellent agreement with Fig. 3. Once again, qualitative agreement is found between the experimental data and the TMM calculations.

In the final configuration, sections 1 and 2 are aligned and oriented opposite to sections 3 and 4, simulating a poling period of $24 \mathrm{~cm}$. Since this poling period is close to the $k_{z}(\omega)=0$ crossing point in Fig. 3, we can expect a strong enhancement of the reflected wave near a $\mathrm{SH}$

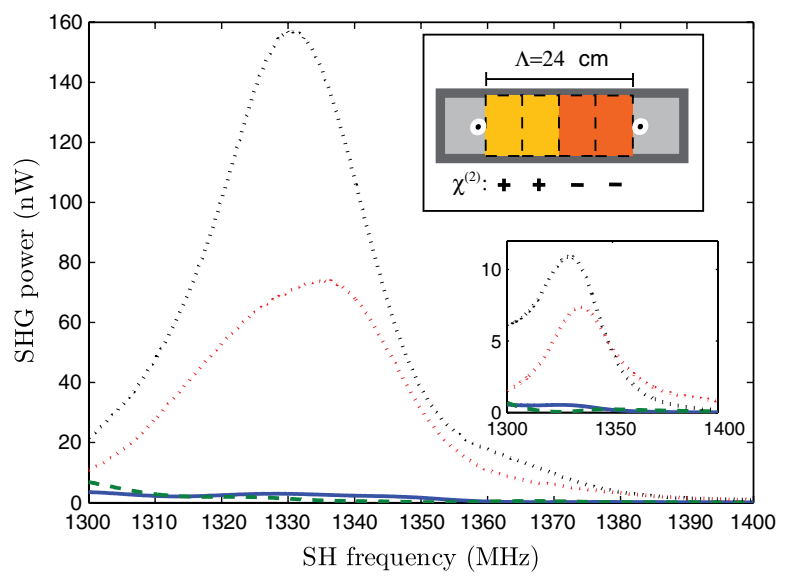

FIG. 7 (color online). Comparison of the transmitted (solid blue and dotted red curves) and reflected (dashed green and dotted black curves) SHG power when the varactors are aligned and when they are periodically poled with $\Lambda=24 \mathrm{~cm}$, respectively. frequency of $1330 \mathrm{MHz}$, with a simultaneous (though weaker) enhancement of the transmitted wave. This is precisely what is shown in Fig. 7, with the SHG powers for the unpoled case plotted for comparison. While the inset again shows good qualitative agreement between the TMM and the experimental results, it should be noted that the peak emitted powers are off by an order of magnitude, whereas much better agreement is found in the previous two configurations. This is most likely due to the inherent sensitivity when approaching the near-zero frequency band, and it was confirmed in TMM calculations (not shown) that small changes in the linear properties produce large changes in the magnitude of the emitted radiation but with similar qualitative behavior. Moreover, it has been shown that spatial dispersion, neglected here, can play an important role as the wave vector vanishes [20].

The exotic PM effects shown here are made possible due to the unique linear properties accessible in NLMMs, in particular, negative- and zero-index behavior. In addition, the use of varying periods to achieve periodic poling to switch between the various SHG schemes demonstrates the compatibility between NLMMs and QPM-a key result. We expect these results to be a necessary and vital step towards the realization of high efficiency nonlinear mirrors and other NLMM-based devices at microwave and terahertz frequencies.

This work was supported by the Air Force Office of Scientific Research (Contract No. FA9550-09-1-0562).

[1] J. B. Pendry et al., IEEE Trans. Microwave Theory Tech. 47, 2075 (1999).

[2] A. A. Zharov, I. V. Shadrivov, and Y. S. Kivshar, Phys. Rev. Lett. 91, 037401 (2003).

[3] M. W. Klein et al., Science 313, 502 (2006).

[4] I. V. Shadrivov et al., Appl. Phys. Lett. 93, 161903 (2008).

[5] D. Huang et al., Appl. Phys. Lett. 98, 204102 (2011).

[6] A. Popov and V. Shalaev, Appl. Phys. B 84, 131 (2006).

[7] E. Poutrina, S. Larouche, and D. R. Smith, Opt. Commun. 283, 1640 (2010).

[8] I. V. Shadrivov et al., Opt. Express 16, 20266 (2008).

[9] B. Wang et al., Opt. Express 16, 16058 (2008).

[10] V. M. Agranovich et al., Phys. Rev. B 69, 165112 (2004).

[11] I. V. Shadrivov, A. A. Zharov, and Y. S. Kivshar, J. Opt. Soc. Am. B 23, 529 (2006).

[12] Skyworks, SMV123x Series: Hyperabrupt Junction Tuning Varactors (data sheet), 2009.

[13] V. G. Veselago, Sov. Phys. Usp. 10, 509 (1968).

[14] D. R. Smith et al., Phys. Rev. Lett. 84, 4184 (2000).

[15] R. Marqués et al., Phys. Rev. Lett. 89, 183901 (2002).

[16] S. Larouche et al., Appl. Phys. Lett. 97, 011109 (2010).

[17] S. Larouche and D. R. Smith, Opt. Commun. 283, 1621 (2010).

[18] A. Rose et al., Phys. Rev. E 82, 036608 (2010).

[19] E. Poutrina, D. Huang, and D. R. Smith, New J. Phys. 12, 093010 (2010).

[20] P. A. Belov et al., Phys. Rev. B 67, 113103 (2003). 